\title{
Bifidobacterium lactis/Lactobacillus acidophilus/L. plantarum/L. salivarius Probiotic Supplement
}

National Cancer Institute

\section{Source}

National Cancer Institute. Bifidobacterium lactis/Lactobacillus acidophilus/L. plantarum/L. salivarius Probiotic Supplement. NCI Thesaurus. Code C114288.

An orally available, probiotic supplement containing the non-pathogenic microorganisms Lactobacillus acidophilus, L. plantarum, L. salivarius and Bifidobacterium lactis, with potential anti-inflammatory, immunomodulating and protective activities. Upon oral ingestion, the naturally-occurring bacterial components in this dietary supplement may improve digestion and help maintain adequate colonization of the gastrointestinal (GI) tract by modulating the composition of the normal microflora. During colonization in the Gl tract, the bacteria may form a protective intestinal barrier that may prevent both damage to the mucosal epithelia caused by toxins and attachment of potential pathogens, which protects against bacterial translocation and infections. In addition, this agent may both reduce the secretion of pro-inflammatory cytokines, including interleukin-10, and potentiate natural and acquired immunity. 